Solís-Marín, F.A., Sandino, O.B., Conejeros-Vargas,

C.A., Caballero-Ochoa, A.A. (2021). Primer registro del género Leptopentacta (Cucumariidae: Colochirinae) en el Caribe nicaragüense. Revista de Biología Tropical, 69(S1), 80-88. DOI 10.15517/rbt. v69iSuppl.1.46329

DOI 10.15517/rbt.v69iSuppl.1.46329

\title{
Primer registro del género Leptopentacta (Cucumariidae: Colochirinae) en el Caribe nicaragüense
}

Francisco Alonso Solís-Marín ${ }^{1 *}$

Osmar Benito Sandino ${ }^{2}$

Carlos Andrés Conejeros-Vargas ${ }^{3}$

Andrea Alejandra Caballero-Ochoa ${ }^{4,5}$

1. Colección Nacional de Equinodermos "Dra. Ma. Elena Caso Muñoz”, Laboratorio de Sistemática y Ecología de Equinodermos, Instituto de Ciencias del Mar y Limnología, Universidad Nacional Autónoma de México, C.P. 04510, Ciudad de México, México; fasolis@cmarl.unam.mx (*Correspondencia).

2. Departamento de Biología. Edificio de Ciencias Básicas, Universidad Nacional Autónoma de Nicaragua-León. C.P. 21000, León, Nicaragua; sandino_lucas08@yahoo.es

3. Posgrado en Ciencias del Mar y Limnología, Universidad Nacional Autónoma de México, Av. Ciudad Universitaria 3000, C.P. 04510, Coyoacán, Ciudad de México, México; conejeros@ciencias.unam.mx

4. Facultad de Ciencias, Universidad Nacional Autónoma de México, Circuito exterior s/n, C.P. 04510, Ciudad de México, México; a.caballero.ochoa@ciencias.unam.mx

5. Posgrado en Ciencias Biológicas, Universidad Nacional Autónoma de México, Av. Ciudad Universitaria 3000, C.P. 04510, Coyoacán, Ciudad de México, México.

Recibido 10-VI-2020. Corregido 18-VII-2020. Aceptado 17-VIII-2020.

\section{ABSTRACT}

First record of the genus Leptopentacta (Cucumariidae: Colochirinae) for the Nicaraguan Caribbean.

Introduction: The genus Leptopentacta has only been previously reported from Florida, Cuba, Colombia, Costa Rica, and Suriname. Objective: To present the first record of the genus for Nicaragua. Methods: The morphological characters of Nicaraguan material at the Smithsonian Institution (USNM 1014529), were corroborated with the original description. Results: The five specimens collected in the Nicaraguan Caribbean match the genus. Conclusions: Leptopentacta is reported for the first time from the Nicaraguan Caribbean Sea. This increases the number of Nicaraguan echinoderms to 194 species.

Key words: Holothuroidea; Atlantic; sea cucumber; taxonomy; morphology.

En el último trabajo donde se recopiló la información correspondiente a la biodiversidad de equinodermos para la costa de Nicaragua se reportó un total de 193 especies distribuidas en las cinco clases, particularmente para el Caribe nicaragüense se tienen reportadas 21 especies de holoturoideos que representan poco más del $15 \%$ de las 138 especies reportadas de esta clase para la zona (Sandino et al., 2017).
El género Leptopentacta fue descrito por Clark (1938) para agrupar a los pepinos de mar con: 1) cuerpo alargado, pentagonal y más o menos curvado con una pared corporal firme, la cual se caracteriza por la presencia de osículos en forma de grandes escamas o pequeñas placas cubiertas por una epidermis calcificada, 2) con los podios dispuestos en una sola hilera recta o en zigzag en cada una de 
las áreas ambulacrales, siendo más abundantes y robustos aquellos dispuestos en la superficie ventral en comparación a las áreas ambulacrales de la superficie dorsal, y 3) corona con 10 tentáculos, siempre el par más ventral de menor tamaño. En este género actualmente se tienen incluidas 13 especies (WoRMS, 2020), las cuales se distribuyen: tres en el Indo Pacífico, una en el Pacífico Noreste, tres en el Pacífico Este tropical, una en el Atlántico Norte, una en el Golfo de Bengala (océano Índico), dos en el Mediterráneo, una en el Mar Negro y una en el Caribe; distribuidas batimétricamente de los 0 a los $243 \mathrm{~m}$. De las 13 especies antes mencionadas, la que se distribuye en el Caribe es Leptopentacta deichmannae Domantay, 1958 (Domantay, 1958; González, Solano, \& Navas, 2002; Alvarado, 2011; Borrero-Pérez, Benavides-Serrato, \& Diaz-Sanchez, 2012; Lodeiros et al., 2013; Alvarado et al., 2017), con un rango batimétrico de los 0 a los $37 \mathrm{~m}$ (Domantay, 1958; Solís-Marín et al., 2013).

El objetivo del presente trabajo es presentar el primer registro del género Leptopentacta para la costa del Mar Caribe nicaragüense obtenido al examinar el material depositado en la Colección Científica del National Museum of Natural History, Smithsonian Institution, Washington, D.C., E.U.A. (USNM).

\section{MATERIALES Y MÉTODOS}

Dentro del material incluido en este trabajo se revisaron los especímenes contenidos en el lote USNM 1014529 (National Museum of Natural History, Smithsonian Institution, Washington D.C., E.U.A.). Se realizó una búsqueda bibliográfica de los trabajos que reportaran especies pertenecientes al género Leptopentacta, así como de los trabajos en los que se describen su morfología. El análisis de la morfología de los ejemplares se llevó a cabo en el Laboratorio de Sistemática y Ecología de Equinodermos del Instituto de Ciencias del Mar y Limnología de la Universidad Nacional Autónoma de México (ICML-UNAM) con ayuda de un microscopio estereoscópico Olympus SZ5. La toma de fotografías multifocales del organismo completo y del anillo calcáreo se realizó con un microscopio estereoscópico Leica Z16 APOA con una cámara Leica DFC490; para unir las fotografías se utilizó el programa Leica Application Suite (LAS) versión 4.3.0. Este procedimiento se realizó en el Laboratorio de la Biodiversidad II del Instituto de Biología, Universidad Nacional Autónoma de México. Para el análisis de las espículas, se extrajeron osículos de la pared del cuerpo (región anterior, media y posterior) y de los tentáculos (base y ramificación). El tejido se disolvió en lejía doméstica fresca (5-6.5 \%) en tubos de centrífuga. Después de la centrifugación a $1000 \mathrm{rpm}$ durante $10 \mathrm{~min}$, el blanqueador se pipeteó y las espículas se enjuagaron y centrifugaron con agua destilada que se pipeteó posteriormente. El mismo proceso se realizó con concentraciones a 70, 80 y $95 \%$ de etanol. Se añadió etanol absoluto a las espículas y finalmente se tomó una pequeña alícuota y se colocó para secar en un trozo cilíndrico de cinta de carbono conductora de doble recubrimiento. La muestra se recubrió con oro de $2,5 \mathrm{kV}$ en el ionizador Polaron E3000 durante 3 min y se fotografió usando un microscopio electrónico de barrido Hitachi S-2460N.

Abreviaturas: INVEMAR: Instituto de Investigaciones Marinas y Costeras José Benito Vives de Andréis, Santa Marta, Colombia. INV EQU: Colección de equinodermos del Museo de Historia Natural Marina de Colombia del INVEMAR. LACM: Los Angeles County Museum, Los Angeles, California, E.U.A. USNM: National Museum of Natural History, Smithsonian Institution, Washington, D.C., E.U.A.

\section{RESULTADOS}

Se examinó el lote USNM 1014529, que contiene cinco ejemplares de Leptopentacta sp., los cuales fueron recolectados al Sur de Pearl Cay Point, en el Mar Caribe $\left(12^{\circ} 15^{\prime} 47.81^{\prime \prime} \mathrm{N} \& 83^{\circ} 31^{\prime} 05.86^{\prime}\right.$ "W), por el Pillsbury R/V, St. 7101, el 28 de enero de 1971 a 11-13 m de profundidad. Este constituye el 
primer registro del género Leptopentacta para el Caribe nicaragüense.

\section{Sistemática:}

Orden Dendrochirotida Grube, 1840

Familia Cucumariidae Ludwig, 1894

Subfamilia Colochirinae Panning, 1949

Género Leptopentacta Clark, 1938

Diagnosis (modificada de Clark, 1938 y Deichmann, 1941): Cuerpo alargado, delgado, pentagonal, más o menos curvo. Piel rígida de apariencia reticulada, formada por osículos en forma de escamas grandes o placas pequeñas, cubierto por una epidermis muy calcificada. Pies ambulacrales rígidos llenos de espículas, en una sola serie recta o en zigzag en cada ángulo dorsolateral, y en series dobles más o menos densos en cada ambulacro ventral. Hacia los extremos oral y anal, los pies ambulacrales están dispersos y son papiliformes, a menudo forman cinco valvas terminales. Diez tentáculos, el par ventral es más pequeño y a veces es ausente. Anillo calcáreo simple o con prolongaciones posteriores cortas. Músculos retractores cortos, musculatura longitudinal débilmente desarrollada. Gónadas con pocos tubos en cada mechón, adheridos cerca de la mitad del cuerpo. Espículas: cuerpos reticulados, canastas, de mayor o menor tamaño; escamas o granos reticulados y botones abollonados de cuatro agujeros. Pies con o sin placa terminal, con o sin barrotes de soporte. Tentáculos con barrotes grandes o placas perforadas, a veces también rosetas y barrotes más pequeños.

\section{Leptopentacta sp.}

(Fig. 1A, Fig. 1B, Fig. 1C, Fig. 2A, Fig. 2B, Fig. 2C, Fig. 2D, Fig. 2E, Fig. 2F)

Descripción: tamaño pequeño a mediano (25 a $40 \mathrm{~mm}$ de longitud). Cuerpo pentagonal con ambos extremos dirigidos hacia la superficie dorsal, las regiones terminales del cuerpo (anterior y posterior) se encuentran reducidas en su diámetro dando un aspecto de media luna o con forma de "U" al cuerpo, en algunos organismos la región posterior es más estrecha que la anterior. Boca y ano en posiciones opuestas terminalmente. Corona de tentáculos retraída (Fig. 1A). A lo largo de cada área ambulacral hay una serie única de podios cónicos prominentes que aparentemente representan los pies ambulacrales; los podios de las áreas ambulacrales sobre la superficie central-ventral del cuerpo son de mayor tamaño en comparación a los de las regiones terminales del cuerpo. Sobre las áreas interambulacrales no se observan podios, el tegumento en esta área es rígido, con grandes placas imbricadas a manera de escamas. Corona de tentáculos con 10 tentáculos dendríticos, los dos ventrales son más pequeños que el resto. Anillo calcáreo robusto sin proyecciones posteriores presentes, las placas radiales e interradiales son casi del mismo tamaño y forma (aprox. de $500 \mu \mathrm{m}$ de ancho) (Fig. 1B).

Pared corporal, sobre todo en la región de las áreas interambulacrales con grandes escamas (Fig. 2A) y placas de menor tamaño (Fig. 2B) en disposición reticular, externamente le dan al cuerpo una apariencia de estructuras con forma de escamas, numerosas placas perforadas en forma de disco de varios tamaños, las placas perforadas de las regiones centrales del cuerpo son de mayor tamaño (300 a $500 \mu \mathrm{m}$ de longitud) en comparación a las placas de las regiones terminales del cuerpo (100 a $250 \mu \mathrm{m}$ de longitud). En toda la pared corporal presenta botones abollonados con los bordes irregulares (Fig. 2C) con tamaños que van de 35 a 70 $\mu \mathrm{m}$, en general presentan cuatro perforaciones irregulares en tamaño y forma, algunas de ellas presentan una proyección irregular que puede estar fusionada en otra región de la espícula principalmente con márgenes lisos. Base de los tentáculos con placas curvas, perforadas de diferentes tamaños (100 a $200 \mu \mathrm{m}$ ) ligeramente curvadas, con los bordes irregulares lisos, y con numerosas perforaciones con diferentes formas y tamaños irregularmente dispuestas (Fig. 2D). Zona terminal y ramificaciones de los tentáculos con pequeños (90 a $150 \mu \mathrm{m}$ ) barrotes perforados irregularmente (Fig. 2E), en comparación a las placas curvas perforadas de la base de los tentáculos. Pared corporal 


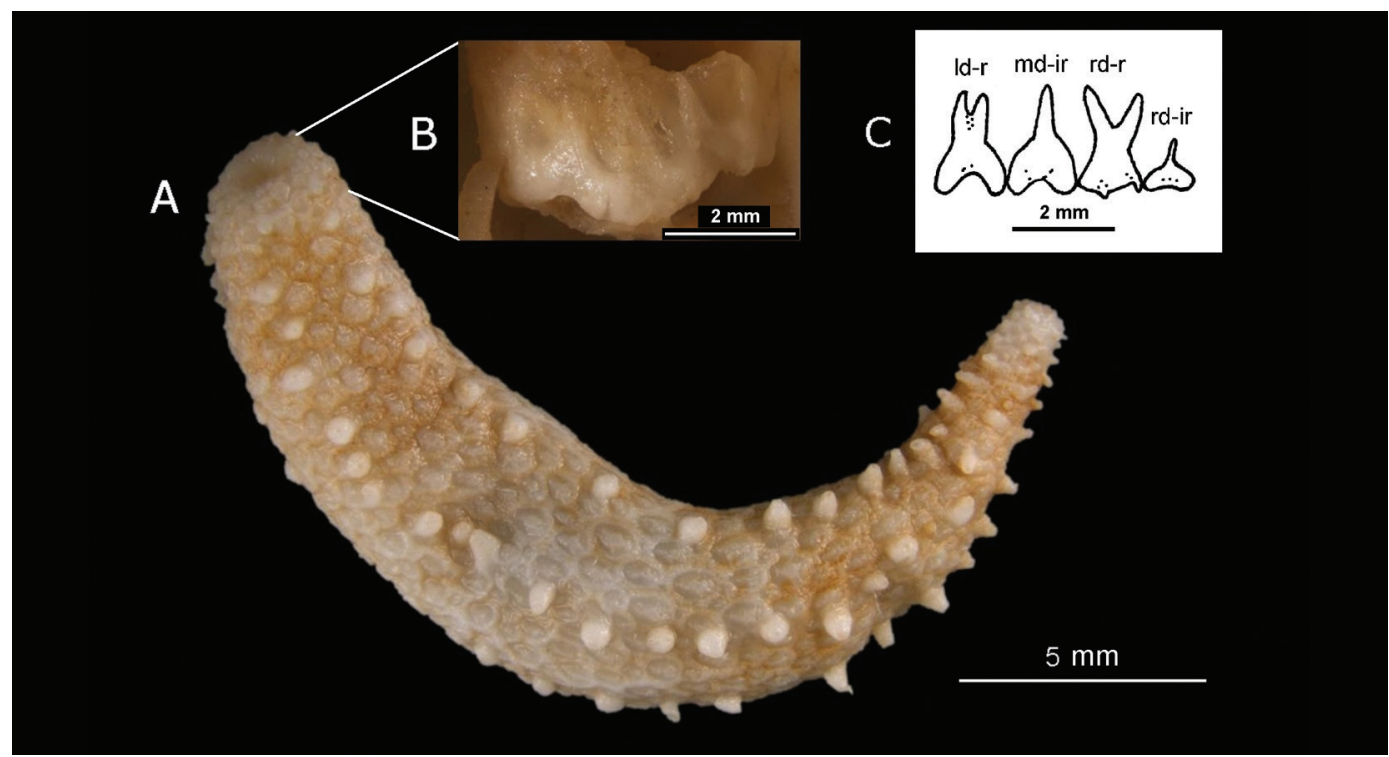

Fig. 1. Leptopentacta sp. USNM 1014529. A. Vista lateral externa. B. Anillo calcáreo. C. Dibujo del anillo calcáreo. Placas ldr = radial dorsal izquierda; md-ir = interadial media dorsal; rd-r = radial dorsal derecha; $r$ d-ir = interadial dorsal derecha. Fig. 1. Leptopentacta sp. USNM 1014529. A. External, lateral view. B. Calcareous ring. C. Drawing of the calcareous ring. Plates ldr = left dorsal radial; md-ir = mid dorsal interradial; rd-r = right dorsal radial; rd-ir $=$ right dorsal inter-radial.

con pequeñas canastas delicadas con forma de mazo o cucharón (40 a $80 \mu \mathrm{m}$ de longitud) (Fig. $2 \mathrm{~F})$, estas estructuras son poco abundantes.

Material examinado: cinco ejemplares, USNM 1014529.

Localidad: al Sur de Pearl Cay Point, en el Mar Caribe $\left(12^{\circ} 15^{\prime} 47.81^{\prime \prime} N \& 83^{\circ} 31^{\prime} 05.86^{\prime}\right.$ " W), por el Pillsbury R/V, St. 7101 (28 de enero de 1971).

Distribución batimétrica: 11-13 m.

Leptopentacta deichmannae Domantay, 1958 (Fig. 3A, Fig. 3B, Fig. 3C, Fig. 3D, Fig. 3E)

Leptopentacta deichmannae Domantay, 1953: 140; Domantay, 1958: 193-194; González et al., 2002: 122-123; Alvarado, 2011: 274; Borrero-Pérez et al., 2012: 155-156; Lodeiros et al., 2013: 247; Solís-Marín et al., 2013: 646: Alvarado et al., 2017: S281.

Diagnosis (modificada de Domantay, 1958 y Borrero-Pérez et al., 2012): Organismos delgados, de talla pequeña a mediana, pueden alcanzar una longitud de $50 \mathrm{~mm}$. Cuerpo con forma pentagonal en corte transversal, con extremos curvos hacia la superficie dorsal, dando una apariencia de media luna. Boca y ano terminales. Apéndices cónicos prominentes a lo largo de cada área ambulacral. Espículas con forma de escamas reticuladas e imbricadas, dispuestas irregularmente en la superficie del cuerpo. Además de estas escamas, la pared del cuerpo presenta numerosas placas con forma de disco, perforadas con el borde liso; también presenta canastas pequeñas y espículas con forma de matraz. Los apéndices cónicos son conspicuos y poco numerosos en relación con las demás especies de su género.

Material tipo: Sintipos, LACM E.1939177.5, LACM E.1939-174.6. Con base en el Código Internacional de Nomenclatura Zoológica (artículo 73.2), "cuando el autor no designa material de la serie tipo, todos los ejemplares de la serie tipo son sintipos automáticamente si no se han designado holotipo ni lectotipo" (Comisión Internacional de Nomenclatura Zoológica, 1999).

Localidad tipo: Florida (Domantay, 1958).

Distribución geográfica: Florida (E.U.A), Cuba, Costa Rica, Colombia, Venezuela y 


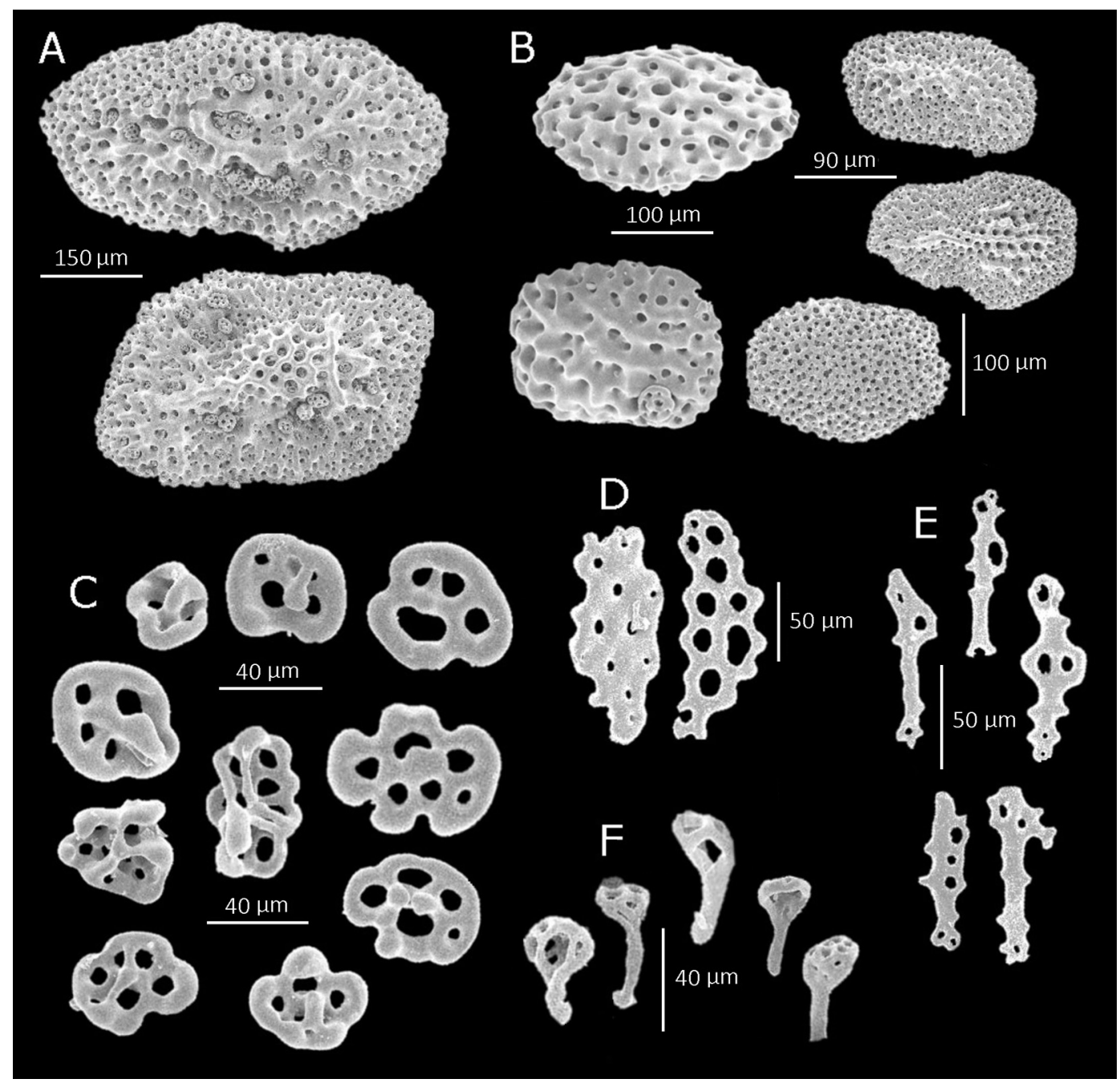

Fig. 2. Leptopentacta sp. USNM 1014529. A. Osículos en forma de escamas de la pared del cuerpo. B. Placas perforadas de la pared del cuerpo (áreas interambulacrales). C. Botones abollonados irregulares de la pared del cuerpo. D. Placas curvas perforadas de la base de los tentáculos. E. Barrotes perforados de las ramificaciones de los tentáculos. F. Pequeñas canastas con forma de mazo o cucharón de la pared del cuerpo.

Fig. 2. Leptopentacta sp. USNM 1014529. A. Scales ossicles from body wall. B. Perforated plates from body wall (interambulatory areas). C. Irregular knobbed buttons from body wall. D. Perforated curved plates at the base of the tentacles. E. Perforated bars of the ramifications of the tentacles. F. Small mallet or ladle-shape baskets from body wall.

Surinam (Domantay, 1953, 1958; González et al., 2002; Alvarado \& Solís-Marín, 2013; Borrero-Pérez et al., 2012).

Distribución batimétrica: De 0 a $37 \mathrm{~m}$ (Domantay, 1958).

Notas ecológicas: L. deichmannae habita en fondo lodoso, material vegetal, fragmentos de conchas y esponjas (Domantay,
1958; González et al., 2002; Borrero-Pérez et al., 2012).

Comentarios: L. deichmannae se considera "nomen nudum" dado que fue nombrada por Domantay (1953) sin brindar una diagnosis. Aunque años después Domantay (1958) la describe formalmente, la Comisión Internacional de Nomenclatura Zoológica (1999) establece 


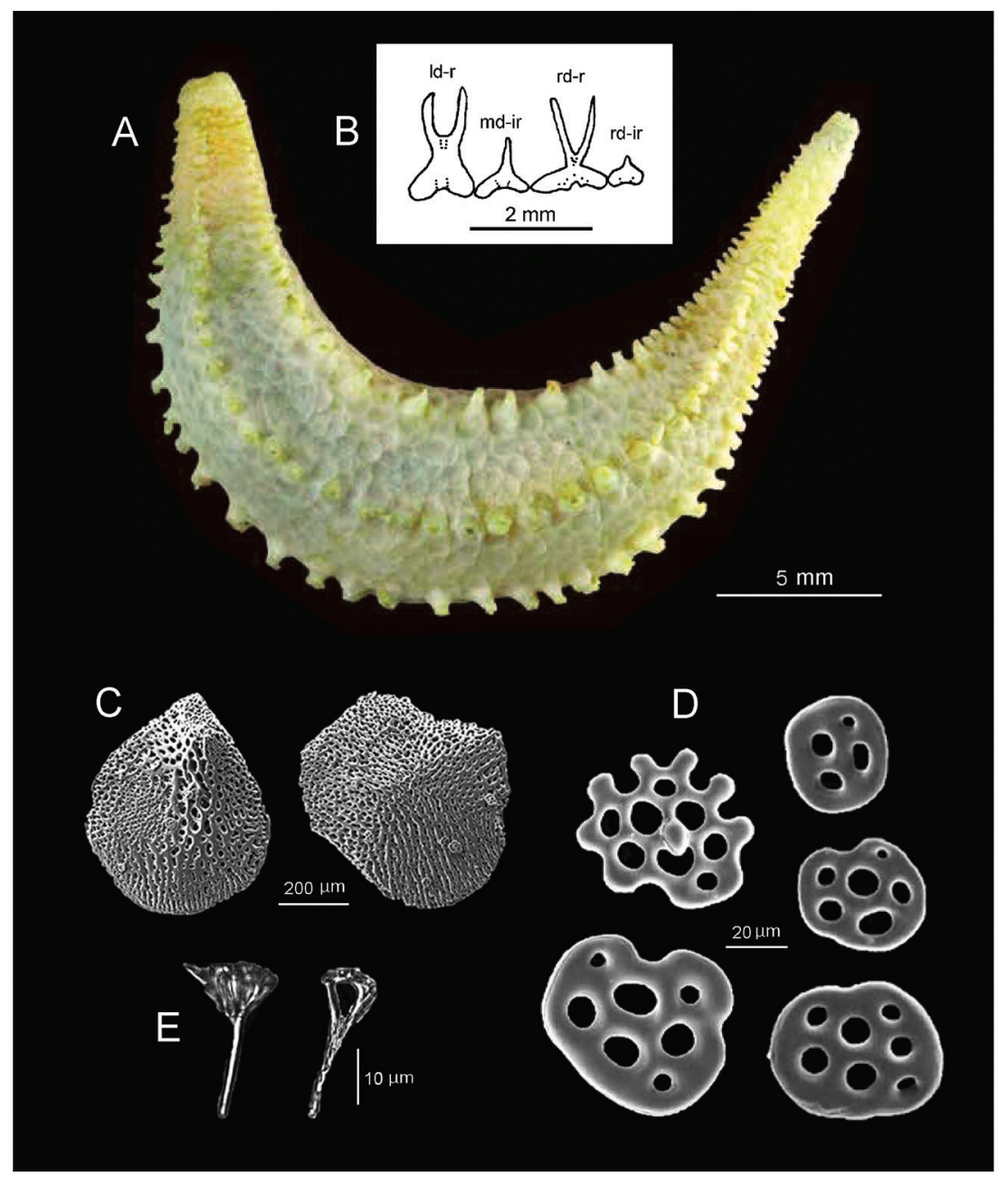

Fig. 3. Leptopentacta deichmannae Domantay, 1953. INV EQU 1353. A. Vista lateral externa. B. Dibujo del anillo calcáreo. C. Osículos en forma de escamas imbricadas de la pared corporal. D. Placas perforadas de la pared corporal. E. Canastas en forma de matraz. Placas ldr = radial dorsal izquierda; md-ir = interadial media dorsal; rd-r = radial dorsal derecha; rd-ir = interadial dorsal derecha. Modificado de Borrero-Pérez et al. (2012).

Fig. 3. Leptopentacta deichmannae Domantay, 1953. INV EQU 1353. A. External, lateral view. B. Drawing of the calcareous ring. C. Imbricated scales ossicles of the body wall. D. Perforated plates of the body wall. E. Flask-shaped baskets. Plates ldr = left dorsal radial; $\mathrm{md}-\mathrm{ir}=$ mid dorsal interradial; $\mathrm{rd}-\mathrm{r}=$ right dorsal radial; $\mathrm{rd}$-ir $=$ right dorsal interradial. Modified from Borrero-Pérez et al. (2012).

que sea tratado como un nombre desnudo (artículo 13). Esta especie también es considerada "nomen dubium" ya que desde su descripción formal (Domantay, 1958) presenta un diagnóstico insuficiente (e.g. carece de ilustraciones) y no se nombró a los ejemplares tipo.

\section{DISCUSIÓN}

Al momento de analizar los ejemplares depositados bajo el número de catálogo USNM 1014529, nos percatamos que el material había sido identificado previamente como Ocnus sp. 
esto quizá debido a la presencia de botones abollonados y de canastas en la pared corporal; la diferencia entre estos géneros es la forma de los botones abollonados y las canastas, los cuales tienden a ser generalmente de forma irregular en el género Leptopentacta (Miller \& Pawson, 1984).

La morfología externa e interna de los ejemplares de Leptopentacta sp. se parece a la de Leptopentacta deichmannae Domantay, 1958 (nomen nudum, nomen dubium), única especie del género reportada para el gran Caribe (Domantay, 1953; Domantay, 1958; González et al., 2002; Borrero-Pérez et al., 2012; Alvarado \& Solís-Marín, 2013). En ambas especies se puede identificar las bandas de podios únicamente sobre las cinco regiones ambulacrales y las áreas interambulacrales libres; sin embargo, los ejemplares de $L$. deichmannae tienen un aspecto pentagonal con las regiones terminales reducidas y con un número de podios mayor y de menor tamaño en comparación a la región central del cuerpo (Fig. 3A) mientras que en los ejemplares de Leptopentacta sp. son más cilíndricos, la región anterior es más grande que la posterior y los podios a lo largo de todo el cuerpo son más robustos y casi del mismo tamaño (Fig. 1A). En las áreas interambulacrales de ambas especies se pueden identificar osículos grandes en forma de escamas, siendo de mayor tamaño las escamas de la región central y reduciéndose en dirección a las regiones terminales del cuerpo (Fig. 1A, 3A); las escamas de L. deichmannae son de mayor tamaño (400-600 $\mu \mathrm{m}$ de ancho), con uno de los extremos ligeramente más afilado, dándole aspecto de gota (Fig. 3C) en comparación a las escamas de Leptopentacta sp. las cuales son de menor tamaño (300-400 $\mu \mathrm{m}$ de ancho) y de apariencia rectangular u ovalada (Fig. 2A). Además de las escamas de la piel, Leptopentacta sp. presenta grandes placas perforadas (Fig. 2B) las cuales se localizan alrededor de las escamas.

Las espículas de la pared corporal con forma de botones lisos con márgenes lisos y planos en su mayoría, reportadas en el trabajo de González et al. (2002; p. 123, Fig. 30) son consideradas e ilustradas en el trabajo de Borrero-Pérez et al. (2012; p. 155, lámina 2, Fig. A) como "placas perforadas con los bordes irregulares o regulares", en donde las formas irregulares son ligeramente parecidas a las formas presentadas en este trabajo (Fig. 2C), pero no tan complejas. Los botones de la pared corporal entre L. deichmannae y Leptopentacta sp. son muy distintos, siendo lisos, con cuatro a siete perforaciones (Fig. 3D) y con borde irregular en la primera, y abollonados, con cuatro a ocho perforaciones y con borde irregular en Leptopentacta sp. (Fig. 2C). Las canastas con forma de mazo o cucharón ilustradas en el presente trabajo (Fig. 2F) presentan distinta morfología y tamaño en comparación a las de Borrero-Pérez et al. (Fig. 3E), quienes las denominan "canastas en forma de matraz"; en Leptopentacta sp. son de 30 a $40 \mu \mathrm{m}$ de largo, mientras que en $L$. deichmannae son más pequeñas $(20 \mu \mathrm{m})$.

Los anillos calcáreos de ambas especies son simples, sin proyecciones posteriores, pero se diferencian en las formas de las placas radiales dorsales derechas, con proyecciones anteriores más delgadas en $L$. deichmannae (Fig. 3B) y más robustas y cortas en Leptopentacta sp. (Fig. 3C).

Se reporta por primera vez la presencia del género Leptopentacta Clark, 1938 dentro de los límites del Mar Caribe nicaragüense. Con este hallazgo, se incrementa el número total de holoturoideos reportados para esta zona a 22 especies y 194 especies de equinodermos para las costas nicaragüenses.

Declaración de ética: los autores declaran que todos están de acuerdo con esta publicación y que han hecho aportes que justifican su autoría; que no hay conflicto de interés de ningún tipo; y que han cumplido con todos los requisitos y procedimientos éticos y legales pertinentes. Todas las fuentes de financiamiento se detallan plena y claramente en la sección de agradecimientos. El respectivo documento legal firmado se encuentra en los archivos de la revista. 


\section{AGRADECIMIENTOS}

Al Dr. David Pawson (USNM) por permitirnos el acceso a los ejemplares mencionados en el presente trabajo. A la Dra. Giomar Borrero Pérez (Instituto de Investigaciones Marinas y Costeras, INVEMAR, Santa Marta Colombia), por su ayuda para la revisión de material alojado en la Colección de equinodermos del Museo de Historia Natural Marina de Colombia del INVEMAR. A Berenit Mendoza Garfias (Laboratorio de Microscopía y Fotografía de la Biodiversidad I, Instituto de Biología, UNAM) por la asesoría y el apoyo en la toma de fotografías de MEB y a Susana Guzmán Gómez (Laboratorio de Microscopía y Fotografía de la Biodiversidad II, Instituto de Biología, UNAM) por la toma de fotografías multifocales empleadas para ilustrar el presente trabajo. A Alicia Durán González y a Ma. Esther Diupotex del ICML, UNAM por las labores técnicas realizadas en el desarrollo de este trabajo. CACV (becario 666781) agradece al Consejo Nacional de Ciencia y Tecnología (CONACyT) por la beca de doctorado 722925 .

\section{RESUMEN}

\section{Primer registro del género Leptopentacta (Cucumariidae: Colochirinae) en el Caribe nicaragüense}

Introducción: El género Leptopentacta solo ha sido reportado previamente en Florida, Cuba, Colombia, Costa Rica y Surinam. Objetivo: Presentar el primer registro del género para Nicaragua. Métodos: Los caracteres morfológicos del material nicaragüense del Smithsonian Institution (USNM 1014529), fueron corroborados con la descripción original. Resultados: Los cinco especímenes recolectados en el Caribe nicaragüense coinciden con el género. Conclusiones: Leptopentacta se reporta por primera vez en el Mar Caribe de Nicaragua. Esto aumenta el número de equinodermos nicaragüenses a 194 especies.

Palabras clave: Holothuroidea; Atlántico; pepino de mar; taxonomía; morfología.

\section{REFERENCIAS}

Alvarado, J.J. (2011). Echinoderm diversity in the Caribbean Sea. Marine Biodiversity, 41, 261-285.
Alvarado, J.J., \& Solís-Marín, F.A. (2013). Echinoderm Research and Diversity in Latin America. Berlin, Heidelberg: Springer.

Alvarado, J.J., Chacón-Monge, J.L., Solís-Marín, F.A., Pineda-Enríquez, T., Caballero-Ochoa, A.A., SolanoRivera, S., \& Chaves, R.R. (2017). Equinodermos del Museo de Zoología de la Universidad de Costa Rica. Revista de Biología Tropical, 65(1), S272-S287.

Borrero-Pérez, G.H., Benavides-Serrato, M., \& DiazSanchez, C.M. (2012). Equinodermos del Caribe colombiano II: Echinoidea y Holothuroidea. Santa Marta, Colombia: Serie de Publicaciones Especiales de Invemar.

Clark, H.L. (1938). Echinoderms from Australia, an account of collections made in 1929 and 1932. Memoirs of the Museum of Comparative Zöology at Harvard College, 55, 1-597.

Comisión Internacional de Nomenclatura Zoológica. (1999). Código Internacional de Nomenclatura Zoológica (4 ${ }^{\mathrm{a}}$ Ed.). Londres, Inglaterra: International Trust of Zoological Nomenclature.

Deichmann, E. (1941). The Holothurioidea collected by the Velero III during the years 1932 to 1938. Part I, Dendrochirota. Allan Hancock Pacific Expeditions, $8,61-153$.

Domantay, J.S. (1953). A brief summary of the Pacific and Atlantic Holothuroidea of the Allan Hancock Foundation Collections. Philippine Journal of Science, $82,133-140$.

Domantay, J.S. (1958). Some holothurians from Florida in the collections of the Allan Hancock Foundation. Agra University journal of research. Science, 7(2), 181-202.

González, D.N., Solano, O.D., \& Navas, G. (2002). Equinodermos colectados por la expedición $\mathrm{CIOH}-$ INVEMAR-SMITHSONIAN desde Cartagena hasta el Golfo de Urabá, Caribe colombiano. Boletín de Investigaciones Marinas y Costeras, 31, 85-132.

Grube, A.E. (1840). Actinien, Echinodermen und Würmer des Adriatischen- und Mittelmeers nach eigenen Sammlungen beschrieben. Königsberg: J.H. Bon.

Lodeiros, C., Martín, A., Francisco, V., Noriega, N., Díaz, Y., Reyes, J., Aguilera, O., \& Alió, J. (2013). Echinoderms from Venezuela: Scientific Recount, Diversity and Distribution. En J.J. Alvarado, \& F.A. Solís-Marín (Eds.), Echinoderm Research and Diversity in Latin America (pp. 235-275). Berlin, Heidelberg: Springer.

Ludwig, H. (1894). The Holothurioidea. Reports on an exploration off the west coasts of Mexico, Central and South America, and off the Galapagos Islands, in charge of Alexander Agassiz, by the U. S. Fish Commission Steamer "Albatross," during 1891, Lieut. 
Commander Z. L. Tanner, U. S. N., commanding. XII. Memoirs of the Museum of Comparative Zoölogy at Harvard College, 17(3), 1-183.

Miller, J.E., \& Pawson, D.L. (1984). Holothurians (Echinodermata: Holothuroidea). Memoirs of the Hourglass Cruises, 7(1), 1-79.

Panning, A. (1949). Versuch einer Neuordnung der Familie Cucumariidae (Holothurioidea, Dendrochirotida). Zoologische Jahrbücher. Abteilung für Systematik, Geographie und Biologie der Tiere, 78(4), 404-470.

Sandino, O.B., Solís-Marín, F.A., Caballero-Ochoa, A.A., Conejeros-Vargas, C.A., Márquez-Borrás, F., AyalaAguilera, A.P., \& Laguarda-Figueras, A. (2017).
Equinodermos de Nicaragua: nuevos registros del Pacífico y Caribe Sur. Revista de Biología Tropical, 65(1), S288-S298.

Solís-Marín, F.A., Alvarado, J.J., Abreu-Pérez, M., Aguilera, O., Alió, J., Bacallado-Aránega, ... Williams, S.M. (2013). Appendix: Taxonomic list of the Echinoderms of the Atlantic Ocean and Caribbean Sea of Latin America and the Canary Islands. En J.J. Alvarado \& F.A. Solís-Marín (Eds.), Echinoderm Research and Diversity in Latin America (pp. 601-654). Berlin, Heidelberg: Springer.

WoRMS. (2020). Leptopentacta Clark H.L., 1938. Recuperado de http://www.marinespecies.org/aphia. php? $=$ taxdetails\&id $=123481$ 